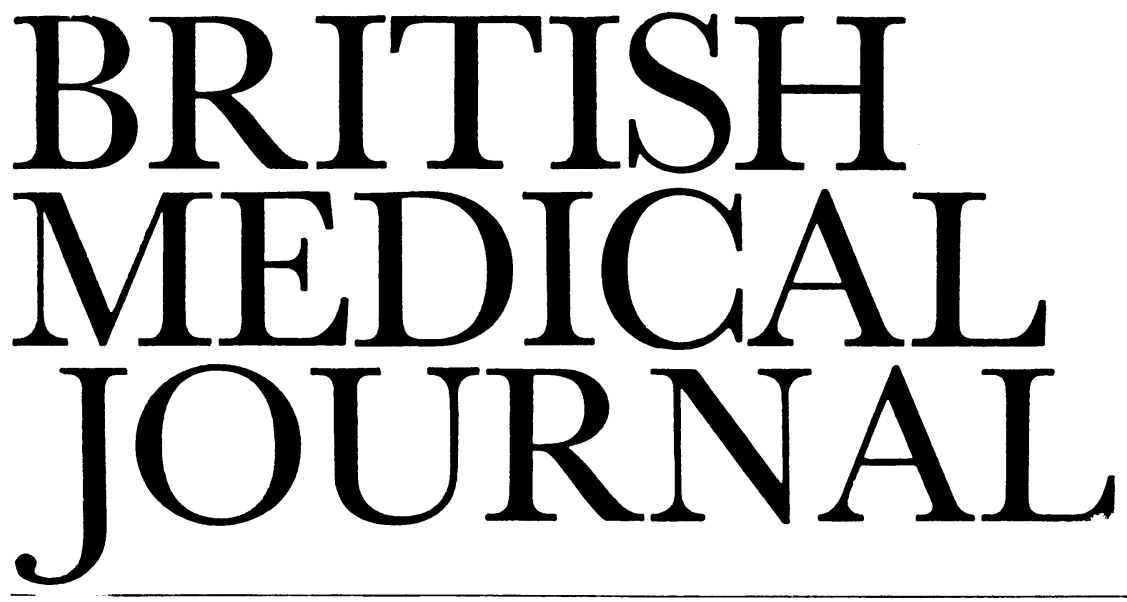

LONDON, SATURDAY 24 MARCH 1984

\title{
Postmarketing surveillance of adverse reactions to drugs
}

Three highly publicised outbreaks of iatrogenic disease have affected Britain in the past 20 years. The thalidomide affair resulted in legal controls on the investigation and marketing of new pharmaceutical products; the practolol episode produced a prolonged period of introspection but no further legislative action; and the problems with benoxaprofen should herald a re-evaluation of our current approaches to postmarketing surveillance of adverse reactions.

In the past techniques such as individual case reports, ${ }^{1}$ the Committee on the Safety of Medicines' "yellow card" system, ${ }^{2}$ monitoring national mortality statistics, ${ }^{3}$ and case-control studies ${ }^{4}$ have successfully identified adverse drug reactions. But the most powerful method, where feasible, remains the cohort investigation, which was used to such effect in the Royal College of General Practitioners' oral contraception study. ${ }^{5}$ In the aftermath of practolol several proposals were made independently for a cohort approach to the identification and quantification of adverse reactions to newly marketed drugs. ${ }^{6-9}$ Though these proposals differed in detail, they all possessed several common features: firstly, that a cohort of patients starting treatment with a particular drug should be identified; secondly, that the cohort should be sufficiently large to allow rarer adverse reactions to be detected; and, thirdly, that the system should look at adverse events (and not merely suspected adverse reactions) suffered by patients from the time of starting treatment to the end of the inquiry.

Several large scale postmarketing studies have now been undertaken specifically to detect adverse drug reactions, and three are of particular interest because of the contrasting methods used. They include a study of cimetidine (with 9928 patients and 9351 controls) by Colin-Jones et al, ${ }^{10}$ an investigation by Allen and Hanburys in 6785 patients starting treatment with labetalol, ${ }^{11}$ and an inquiry by W $\mathrm{H}$ Inman of the Drug Surveillance Research Unit comparing benoxaprofen (11 646 patients) and fenbufen (4113 patients). ${ }^{12} \mathrm{~A}$ fourth study, on 8291 patients given ketotifen, appears at $p 911$.

These four studies adopted different approaches for identifying patients starting treatment. Most patients beginning treatment with cimetidine were identified by dispensing chemists; in the labetalol and ketotifen studies doctors recruited patients themselves; and Inman used the Prescription Pricing Authority to extract prescriptions for benoxaprofen and fenbufen. The three techniques appear to have been satisfactory, though the technique used by the Drug Surveillance Research Unit is limited by the Prescription Pricing Authority's ability to separate only four different drug names at any one time. ${ }^{13}$
Follow up techniques also varied. The investigators of cimetidine personally inspected patients' general practice records, and the labetalol study relied on general practitioners themselves to complete follow up forms at specified times. A similar technique was used in the study of ketotifen ( $p$ 911). The Drug Surveillance Research Unit wrote to doctors whose patients had been identified by the Prescription Pricing Authority asking them to supply details of any clinical "events." Not surprisingly, the cimetidine inquiry was able to follow up the highest proportion of patients (over 98\%) at 12 months. Over a similar time period the completeness of follow up in the labetalol and ketotifen studies was 70\% and $41 \%$ respectively, and $52 \%$ in the benoxaprofen inquiry. In a more recent study by the Drug Surveillance Research Unit of erythromycin and jaundice the response rate was $76 \%{ }^{14}$

All these studies appear to have been reasonably successful on methodological grounds, but they highlight several problems. Firstly, none of the techniques used for recruiting patients was entirely appropriate for postmarketing surveillance in hospitals. This is particularly important for those drugs such as anaesthetic agents and radiological contrast media whose use is confined to hospital practice. Secondly, the studies in which doctors themselves were asked to provide details of their patients yielded incomplete response rates. Though there is no evidence that this resulted in distorted patterns of reporting of adverse events, the possibility of bias in future studies is worrying. Thirdly, the cimetidine study showed how much care is needed in interpreting the results of postmarketing surveillance investigations. A casual inspection of the deaths among cimetidine takers and controls might have suggested an association between the drug and cancer of the stomach and lung, haematological malignancies, and chronic liver disease. Closer inquiry, however, showed that none of these associations could be attributed to the use of cimetidine. The study of benoxaprofen and fenbufen disclosed an undetected adverse effect of the former (irritation of the bladder), showing that the technique can recognise novel adverse drug reactions. Fourthly, in all four studies the follow up was confined to a relatively short time, and I doubt whether any of these methods would be effective over a five to 10 year period.

Two further fundamental issues have yet to be resolved: the problem of numbers and the need for a control population. If an adverse drug reaction occurs with an incidence of one in 1000 some 3000 drug takers need to be studied for a $95 \%$ chance of observing the reaction just once. ${ }^{15}$ At the start of these three studies, only those reactions occurring in more 
than one in 3300 (cimetidine), one in 2300 (labetalol), and one in 3900 (benoxaprofen) cases were likely to have been observed. Because of incomplete follow up of patients receiving labetalol and benoxaprofen, the final tally was about one in 1400 and one in 1900 respectively. This probably explains the failure to detect a significant increase in adverse reactions on the liver associated with benoxaprofen or the lichenoid skin eruptions with labetalol. Indeed, in his Wolfson lecture at Oxford in January 1984, Inman presented preliminary results from a larger $(24000)$ cohort of patients receiving benoxaprofen: of the 54 patients who developed jaundice while taking the drug, there was one (non-fatal) case where treatment could be blamed and a further 11 cases (including six with incomplete follow up) when alternative causes could not be entirely ruled out. Even so, this may underestimate the problem since the duration of treatment with benoxaprofen in Inman's most recent study averaged about seven months while the average duration of treatment in patients with fatal hepatorenal syndrome associated with benoxaprofen reported to the Committee on the Safety of Medicines was 8.5 months. ${ }^{16}$ Increasing the number of patients in postmarketing surveillance studies, as well as including sufficient numbers undergoing prolonged treatment, would enhance the sensitivity of the technique but substantially increase the cost.

The need for controls in postmarketing surveillance studies seems to me to be clear. This is particularly the case when adverse "events" are to be recorded, when the underlying disorder is itself associated with substantial morbidity or mortality (for example, hypertension), or when it is likely that the drug will increase the incidence of commonly occurring symptoms or diseases (for example, impotence or diabetes mellitus). Thus, in the recent ketotifen study, the significance of adverse "events" occurring during follow up (including weight gain and non-fatal myocardial infarction) is impossible to evaluate. Yet the choice of a control population is difficult. The ideal group-patients suffering from the same disease but undergoing no active treatment-is unattainable for long term studies except in very special circumstances. More realistic controls include patients treated with a comparable drug (as in the benoxaprofen study) or a sample of the general population (as in the cimetidine study).

For the future we need to be able to identify the drugs to which these postmarketing surveillance techniques might most profitably be applied. In general, the balance between risk and benefit most needs to be examined with drugs for disorders which are not life threatening and when reasonably safe alternatives are already available. Such studies are likely to be worth while, however, only if they enrol at least 10 times the number of patients in the premarketing trials and include a reasonable comparative control group. They will therefore need to include at least 10000 patients and 10000 controls, and the cost will be substantial. Yet they will detect, with $95 \%$ confidence, only those events occurring in one in $\mathbf{3 0 0 0}$ patients. For many drugs, and for the recognition and confirmation of less common reactions, we must therefore continue to rely on published anecdotal reports, the yellow card system, case-control studies, and examination of mortality and morbidity statistics.

Michael D RaWlins

Professor of Clinical Pharmacology, Wolfson Unit of Clinical Pharmacology, The University,

Newcastle upon Tyne NE1 7RU
1 Venning GR. Identification of adverse reactions to new drugs. III: Alerting processes and early warning systems. $\mathrm{Br}$ Med $\mathcal{F} 1983 ; 286$ : 458-60.

${ }^{2}$ Inman WHW. Monitoring for drug safety: the United Kingdom. In: Inman WHW, ed. Monitoring for drug safety. Lancaster: MTP Press Ltd, 1980:9-47.

${ }^{3}$ Stolley PD. The use of vital and morbidity statistics for the detection of adverse drug reactions and for monitoring of drug safety. $\mathcal{f}$ Clin Pharmacol $1982 ; 22: 499-504$.

4 Mann JI. Principles and pitfalls in drug epidemiology. In : Inman WHW, ed. Monitoring for drug safety. Lancaster: MTP Press Ltd, 1980: 401-15.

5 Royal College of General Practitioners. Oral contraceptives and health. Tunbridge Wells: Pitman Medical, 1974.

${ }^{6}$ Dollery CT, Rawlins MD. Monitoring adverse reactions to drugs. $\mathrm{Br}$ Med F 1977; :96-7.

${ }^{7}$ Inman WHW. Recorded release. In: Gross FH, Inman WHW, eds. Drug monitoring. New York: Academic Press, 1977:65-78.

${ }^{8}$ Lawson DH, Henry DA. Monitoring adverse reactions to new drugs "restricted release" or "monitored release" ? Br Med f 1977;i:691-2.

${ }^{\circ}$ Wilson AB. Postmarketing surveillance of adverse reactions to new medicines. Br Med F 1977; ii:1001-3.

10 Colin-Jones DG, Langman MJS, Lawson DH, Vessey MP. Postmarketing surveillance of the safety of cimetidine: 12 month mortality report. Br Med F 1983;286:1713-6.

11 Glaxo Group Research Registration Department. Monitored release of Trandate in general practice. London: Glaxo Group Research, 1980.

12 Inman WHW. Prescription-event monitoring. A preliminary study of benoxaprofen and fenbufen. Southampton: Drug Surveillance Research Unit, University of Southampton, 1982.

13 Inman WHW. Brand names, approved names, and drug safety. Lancet $1982 ; \mathrm{i}: 1078$.

14 Inman WHW, Rawson NSB. Erythromycin estolate and jaundice. $\mathrm{Br}$ Med F 1983;286:1954-5.

15 Sackett DL, Haynes RB, Gent M, Taylor DW. Compliance. In: Inman WHW, ed. Monitoring for drug safety. Lancaster: MTP Press Ltd, 1980:427-38.

${ }^{16}$ Griffin JP. The advantages and limitations of drug orientated schemes for monitoring adverse drug reactions. In: Walker $S$, Goldberg $A$, eds. Monitoring for adverse drug reactions. Lancaster: MTP Press (in press).

\section{Hypertension in general practice: what is to be done?}

Two publications from the Veterans Administration Cooperative Study Group on Antihypertensive Agents in 1967 and 1970 and the subsequent trial by the National Heart Foundation of Australia provided the evidence that substantial numbers of asymptomatic patients would benefit from reduction of their blood pressure. ${ }^{1-2 a}$ The potential beneficiaries are those with sustained diastolic pressures above $100 \mathrm{~mm} \mathrm{Hg}$ (phase V), who make up at least $7 \%$ of the population between 35 and 65 years old-which in Britain means over a million people. This takes no account of the much larger numbers with diastolic pressures between 90 and $100 \mathrm{~mm} \mathrm{Hg}$ or of the hypertensive elderly, but both these groups are at present the subject of clinical trials.

The formidable problem of identifying and treating a million or more patients was neatly described over a decade ago in the rule of halves. Surveys had shown that in an unscreened population half the hypertensive patients would not have been identified, of those identified only half would have been having treatment, and of those treated half would have been treated inadequately. This rule set a target for education of general practitioners and trainees, who have been repeatedly encouraged to look for hypertensive patients and institute and maintain treatment. Hart showed what could be done in an individual practice as long ago as $1970,{ }^{3}$ and further publications have spelt out the elements of an effective and practicable policy. ${ }^{5}$ The Royal College of General Practitioners, too, has decided that the main direction of growth for the primary medical services in the foreseeable future should be in anticipa- 